\title{
Prophylactic Use of Troxerutin Can Delay the Development of Diabetic Cognitive Dysfunction and Improve the Expression of Nrf2 in the Hippocampus on STZ Diabetic Rats
}

\author{
Songyun Zhang, Lingling Yuan, Lihui Zhang ${ }^{(D}$, Caige Li, and Jie Li \\ The Second Hospital of Hebei Medical University, No. 215 Heping Road (West), Shijiazhuang City, Hebei Province, China \\ Correspondence should be addressed to Lihui Zhang; zhanglihui10510@163.com
}

Received 19 November 2017; Revised 9 February 2018; Accepted 18 March 2018; Published 12 April 2018

Academic Editor: Tauheed Ishrat

Copyright (c) 2018 Songyun Zhang et al. This is an open access article distributed under the Creative Commons Attribution License, which permits unrestricted use, distribution, and reproduction in any medium, provided the original work is properly cited.

\begin{abstract}
Background. With the change in lifestyle and the aging population, the incidence of cognitive dysfunction in diabetes mellitus is rising sharply. Oxidative stress is an important mechanism in the development of diabetic cognitive dysfunction. Nuclear factor E2-related factor 2 (Nrf2) is the core transcription factor of antioxidative stress. Early prevention and treatment of diabetic cognitive dysfunction can reduce the incidence of dementia and improve the quality of life of diabetic patients. Aim. This study was aimed at determining effect of troxerutin on the development of cognitive dysfunction and the expression level of Nrf2 in the hippocampus of streptozotocin (STZ) diabetic rats, when used in the early preventive stage. Methods. An STZ-induced diabetic rat model was established $(n=30)$, and the animals were randomly divided into 2 groups: diabetic control group (DC, $n=15$ ) and diabetic troxerutin intervention group (DT, $n=15$ ). Another 10 normoglycemic rats were put into a normal control group (NC, $n=10)$. While the DT group was injected with troxerutin $(60 \mathrm{mg} / \mathrm{kg})$, the DC group and the NC group were injected with physiological saline for 12 weeks daily. Learning and memory behaviors were tested using the Morris water maze test. The superoxide dismutase (SOD) activity, malondialdehyde (MDA) content, mRNA level, and protein level of Nrf2 were measured. Data were collected and analyzed by the statistical software package SPSS 19.0, which included one-way analysis of variance with completely randomized design. Results. Learning and memory levels were significantly improved in the DT group compared with the DC group. Moreover, in the DT group, the expression level of Nrf2 in the hippocampus was increased, activity of SOD was elevated, and MDA content was decreased. Conclusion. Prophylactic use of troxerutin delays the development of diabetic cognitive dysfunction and increases the expression level of Nrf2 in the hippocampus of STZ diabetic rats.
\end{abstract}

\section{Introduction}

As diabetes mellitus can cause cognitive dysfunction, it has become an important research domain. However, the exact mechanisms of diabetic encephalopathy are yet to be identified. Nevertheless, increasing evidence indicates that oxidative stress is an important mechanism in the development of diabetic cognitive dysfunction. Malondialdehyde (MDA) content and superoxide dismutase (SOD) activity are classic indicators in assessing the balance between oxidative and antioxidant systems. Nrf2 is considered to be the main transcription factor of antioxidative stress $[1,2]$. In addition, Nrf2 is closely related with cognitive dysfunction $[3,4]$.

Troxerutin is a flavonol (a type of flavonoid) or, more accurately, a hydroxyethylrutoside. Troxerutin has been reported to possess strong antioxidant and antiinflammatory properties [5-9]. Importantly, early prevention and treatment of diabetic cognitive dysfunction can reduce the incidence of dementia and improve the quality of life of diabetic patients [3-7]. This study aimed to observe the development of cognitive dysfunction and 
determine the expression levels of Nrf2 in the hippocampus of streptozotocin (STZ) diabetic rats when using troxerutin in the early preventive stage.

\section{Results}

\subsection{Morris Water Maze Test}

2.1.1. Basic Swimming Speed. There was no statistical difference in swimming speed between the three groups (Table 1).

2.1.2. Navigation Test. Repeated measures ANOVA was used to compare the data between each group. The effect of time did not result in significant changes among the groups $(P=0.058)$. The escape latency of the DC group was significantly longer when compared with that of the NC group $(P<0.001)$. This indicated that diabetic rats had cognitive dysfunction after having successfully modeled for 12 weeks. The DT group had a shorter escape latency than the DC group $(P<0.001)$. This suggests that treatment with troxerutin for 12 weeks can reduce cognitive dysfunction. There were no significant differences in escape latency among the NC, DC, and DT groups after the first day (day $1, P=0.256$ ). Compared with the NC group, the escape latency of DC rats was longer during the subsequent 4 days (day $2, P<0.001$; day $3, P<0.001$; day $4, P<0.001$; day 5 , $P<0.001)$. On day 2 , the escape latency of the DC group was not significantly different from that of the DT group $(P=0.061)$. From the third to the fifth day, the escape latency of the DT group was significantly shorter than that of the DC group (day 3-day 5: $P<0.001$ ) (Table 2).

2.1.3. Probe Test. When comparing the number of times the rats crossed to the platform in $60 \mathrm{~s}$, the number of the $\mathrm{NC}$ group was greater than those of the DC group and DT group. Moreover, the number of the DT group was greater than that of the DC group $\left(F_{2,27}=185.33, P<0.001\right)$ (Table 3$)$.

2.2. HE Staining. After observing a series of histopathologic alterations in the hippocampal neurons of the DC group, the number of normal neurons significantly decreased. On the contrary, the majority of the CAl neurons in the DT and NC groups maintained normal morphology, showing integrated structures and being lined up in order. Moreover, there was less neuron depletion in the DT and NC groups compared to the DC group (Figure 1).

2.3. Immunohistochemical Staining. The optical density (OD) value of the Nrf2-positive cells in the hippocampus of rats in the NC group was higher than that of rats in the DC group and the DT group. Compared with the DC group, the OD value of the Nrf2-positive hippocampal cells of rats in the DT group was increased $\left(F_{2,87}=64.67, P<0.001\right)$ (Figure 2$)$.

2.4. Nrf2 mRNA Expression. When compared with the NC group, mRNA expression of Nrf2 decreased in the DC group and the DT group. Moreover, there was a statistically significant difference between the DC group and the DT group, the Nrf2 mRNA level being higher in the latter $\left(F_{2,15}=2815.91\right.$, $P<0.001$ ) (Figure 3(a)).
TABLE 1: The basic swimming speed of the rats in different groups $(\mathrm{cm} / \mathrm{s}, \bar{x}+\mathrm{s})$.

\begin{tabular}{lcc}
\hline Group & $n$ & The swimming speed \\
\hline NC & 10 & $30.67 \pm 2.58$ \\
DC & 10 & $28.32 \pm 1.65^{\triangle}$ \\
DT & 10 & $30.06 \pm 2.58^{\triangle}$ \\
\hline
\end{tabular}

Note: compared to NC, ${ }^{\triangle} P>0.05$; compared to DC, $\wedge^{\wedge}>0.05$.

2.5. Nrf2 Protein Expression. Total Nrf2 protein: The expression level of total Nrf2 protein in the hippocampus of rats in the DC and the DT groups was lower than that of rats in the NC group. Moreover, the total Nrf2 protein expression of the DT group was significantly higher than that of the DC group $\left(F_{2,15}=107.95, P<0.001\right)$ (Figure 3(b)).

Nuclear Nrf2 protein: The expression level of nuclear Nrf2 protein in the hippocampus of rats in groups DC and DT was lower than that of rats in the NC group. Furthermore, the expression level of nuclear Nrf2 protein of rats in the DT group was higher than that of rats in the DC group $\left(F_{2,15}=65.73, P<0.001\right)$ (Figure 3(c)).

2.6. SOD Activity and MDA Content. SOD activity: The SOD activity of the NC group was higher than that of the DC group. There was no statistical significance between groups NC and DT. However, the SOD activity of the DT group was increased compared with that of the DC group $\left(F_{2,15}=\right.$ 5.873, $P=0.013$ ) (Figure 4(a)).

MDA content: The MDA content of the NC group was lower than that of the DC group. There was no statistical significance between the $\mathrm{NC}$ group and the DT group. However, the MDA content of the DT group was decreased compared with that of the DC group $\left(F_{2,15}=\right.$ 7.868, $P=0.005$ ) (Figure $4(\mathrm{~b})$ ).

\section{Discussion}

Modeling is crucial during an experiment, because if an animal model fails, the experimental results will not be reliable.

To successfully develop the rat model of type 1 diabetes mellitus, rats with blood glucose level $\geq 16.7 \mathrm{mmol} / \mathrm{L}$ at $72 \mathrm{~h}$ after an STZ injection $(60 \mathrm{mg} / \mathrm{kg})$ were included in the model.

Rats with blood glucose level $<16.7 \mathrm{mmol} / \mathrm{L}$ were rejected once a week $[10,11]$. According to the test proposed by Wang et al. [12], 72 SD male rats, aged 8 weeks, were used. Type 1 diabetes mellitus rats began to show cognitive dysfunction symptoms after 6 weeks. More importantly, the symptoms became more apparent after 12 weeks. In this study, rat cognitive function was tested via the Morris water maze, and the results demonstrated that type 1 diabetes mellitus rats became cognitively dysfunctional after 12 weeks.

Troxerutin is 3-hydroxy-rutin and soluble in water [13]. Compared to the intragastric administration, intraperitoneal injection has shorter administration time and is easier to operate. A liquid volume equivalent to $3-8 \%$ of animal weight can be absorbed in one hour. This is because a larger area of the peritoneum and clouds of blood vessels are 
TABLE 2: The escape latencies of the rats in different groups in place navigation test $(s, \bar{x}+s)$.

\begin{tabular}{|c|c|c|c|c|c|c|}
\hline Group & $n$ & Day 1 & Day 2 & Day 3 & Day 4 & Day 5 \\
\hline $\mathrm{NC}$ & 10 & $22.08 \pm 12.22$ & $11.01 \pm 4.19$ & $7.82 \pm 3.84$ & $7.52 \pm 3.06$ & $5.81 \pm 2.33$ \\
\hline DC & 10 & $29.22 \pm 14.10^{\triangle}$ & $32.56 \pm 15.66^{\triangle}$ & $28.58 \pm 12.21^{\triangle}$ & $28.72 \pm 3.59^{\triangle}$ & $24.68 \pm 8.02^{\triangle}$ \\
\hline DT & 10 & $25.94 \pm 15.03^{\triangle \boldsymbol{\Lambda}}$ & $25.38 \pm 16.21^{\triangle \boldsymbol{\Lambda}}$ & $16.00 \pm 8.33^{\triangle \boldsymbol{\Delta}}$ & $14.72 \pm 6.29^{\triangle \boldsymbol{\Lambda}}$ & $15.04 \pm 10.20^{\triangle}$ \\
\hline
\end{tabular}

Note: compared to NC, ${ }^{\triangle} P<0.01$; compared to DC, $\triangle^{\wedge} P<0.01$.

TABLE 3: The times of crossing platform in $60 \mathrm{~s}$ in different groups in spatial probe test $(\bar{x}+s)$.

\begin{tabular}{llc}
\hline Group & $n$ & Times of cross the platform area \\
\hline NC & 10 & $16.00 \pm 1.83$ \\
DC & 10 & $3.00 \pm 0.91^{\triangle}$ \\
DT & 10 & $13.00 \pm 1.83^{\triangle \Delta}$ \\
\hline
\end{tabular}

Note: compared to NC, ${ }^{\triangle} P<0.001$; compared to DC, ${ }^{\mathbf{\Delta}} P<0.001$.

exposed, and the peritoneum has a particularly strong lymphatic absorption capacity for the drug. Clinical trials found that troxerutin has a high safety profile, tolerability, and other advantages, even at high doses [13]. Moreover, a previous study [14] confirmed that $150 \mathrm{mg} / \mathrm{kg} / \mathrm{d}$ (ig) is the optimal dose for treatment of cognitive dysfunction of diabetic rats.

Furthermore, the dose administered to the rats was equivalent to that previously administered to mice, and the dose administered intraperitoneally was 0.3 to 0.5 times greater than the dose administered intragastrically.

Therefore, in this experiment, rats in the DT group were injected with $60 \mathrm{mg} / \mathrm{kg} / \mathrm{d}$ (i.e., $150 \times 0.4 \mathrm{mg} / \mathrm{kg} / \mathrm{d}$ ) of troxerutin.

In the present study, the Morris water maze test was proven to be a credible test for cognitive dysfunction related to hippocampal synaptic plasticity [15]. The navigation test was used to test the spatial learning ability of rats, whereas the probe test was used to test their spatial memory. The basis of cognitive dysfunction in pathology is the connecting dysfunction of the prefrontal-striatum-thalamus-temporal lobe, which features the hippocampus and plays an important role [16]. The hippocampus is one of the most important nerve centers in learning and memory. Its structure and function are closely linked to diabetic cognitive dysfunction [17]. SOD activity reflects antioxidant levels, and MDA content reflects oxidative levels; thus, these measurements are frequently used to assess oxidative damage [18]. Our results were consistent with those of other recent studies, in that troxerutin has strong antioxidant and anti-inflammatory properties [19]. An important cellular defense mechanism under oxidative stress is the Nrf2-mediated expression of endogenous antioxidants [20].

In this experiment, hippocampus tissues were stained with HE, and CA1 hippocampal nerve cells of rats in the NC group were eumorphic, structurally integral, and ordered; cells in the DC group were disordered, with shrunken cell bodies and red cytoplasm, and the number of nerve cells with normal morphology was significantly reduced; cells in the DT group were arranged in order, structurally integrated. These results suggest that the hippocampal damage in the DC group is morphological, which affects the cognitive function. The degree of hippocampal impairment in the DT group was lower than that in the DC group; thus, the preventive use of troxerutin could alleviate damage to the hippocampus and reduce cognitive impairment, to a certain extent.

In this experiment, the blood glucose level of rats in the DT group was not significantly different from that of rats in the DC group. This illustrates that troxerutin cannot decrease blood glucose level, which agrees with the results of Geetha et al., who tested 36 rats weighing $25 \sim 30 \mathrm{~g}$ by administrating a high-fat, high-carbohydrate diet. The results suggested that troxerutin reduces body weight, blood pressure, and insulin resistance [21], but does not induce a significant decrease in blood glucose level. In this study, the results of the Morris water maze test proved that the prophylactic use of troxerutin can delay the development of diabetic cognitive dysfunction in STZ diabetic rats. Additionally, the prophylactic use of troxerutin can increase SOD activity in the hippocampus, suggesting that it can improve the antioxidant ability. Both the mRNA and protein expression of Nrf2 were higher in the DT group than in the DC group. This demonstrated that the prophylactic use of troxerutin in rats increases Nrf2 content in the hippocampus. Therefore, troxerutin administered prophylactically delays the development of diabetes-induced cognitive dysfunction in rats by increasing the Nrf2 content in the hippocampus.

\section{Methods}

4.1. Animals and Drug Administration. All procedures involving animals were in accordance with the Guide for the Care and Use of Laboratory Animals. Forty male Sprague-Dawley rats (age 6-8 weeks, from Beijing Vital River Laboratory Animal Technology Co., certificate number $1100195543)$ were randomly divided into a normal control group (NC group, $n=10$ rats) and a diabetic group ( $n=30$ rats). The rats fasted overnight. Then, STZ was dissolved in $0.1 \mathrm{~mol} / \mathrm{L}$ sodium citrate buffer, $\mathrm{pH} 4.4$, and the 30 rats were intraperitoneally injected with STZ citrate buffer $(60 \mathrm{mg} / \mathrm{kg} / \mathrm{d}$, Sigma Company, USA). At 72 hours after the injection, the blood glucose level of the rats was determined via their tail veins using a blood glucose meter. Fasted rats with a blood glucose level above $16.7 \mathrm{mmol} / \mathrm{L}$ were considered to be diabetic and were randomly divided between a diabetic control group (DC group, $n=15$ ) and a diabetic troxerutin intervention group (DT group, $n=15$ ). 


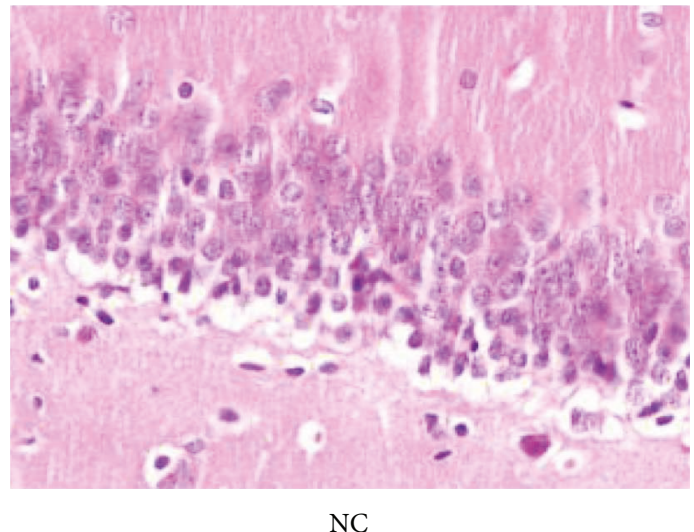

(a)

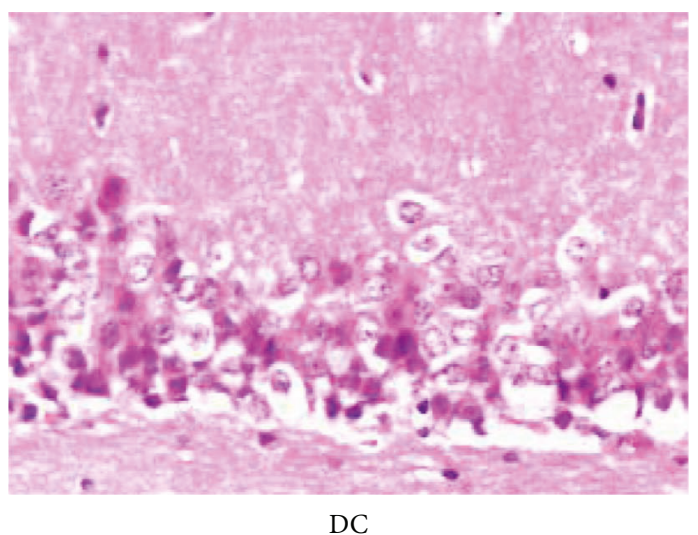

(b)

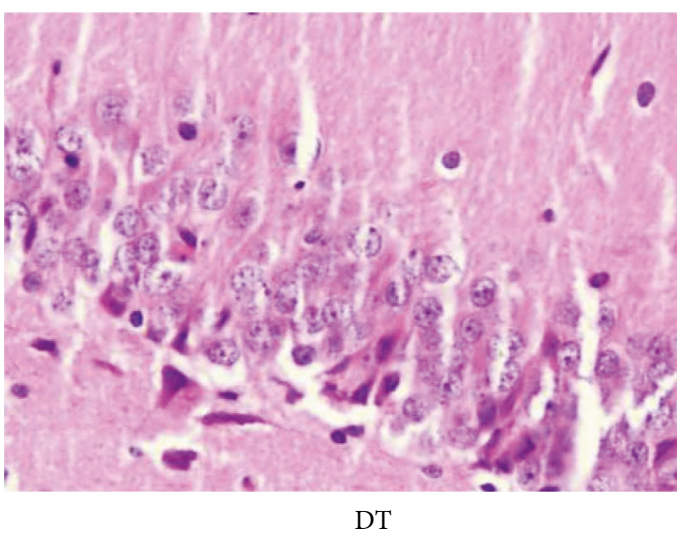

(c)

FIGURE 1: Representative photomicrographs of the hippocampal CA1 area showing the histological changes in different groups (HE, $\times 200$ ). NC: normal control group; DC: diabetic control group; DT: diabetic troxerutin intervention group.

Rats in the DT group were intraperitoneally injected with troxerutin $(60 \mathrm{mg} / \mathrm{kg}, 1 \mathrm{~mL} / \mathrm{kg}$, Jingchun Biochemistry Technology Corporation, Shanghai, China), while those in the DC and NC groups were intraperitoneally injected with physiological saline once daily for 12 weeks. Finally, the blood glucose levels were measured after 12 weeks of troxerutin treatment.

4.2. Morris Water Maze Test. The rats were tested in the Morris water maze at the end of the treatment period (12 weeks). Basic swimming speed of the rats was assessed the day prior to the test. Rats were pretested to determine their treadmill running willingness and those rats which refused to run were excluded from the water maze experiments. The maze consisted of a circular pool $(150 \mathrm{~cm}$ in diameter and $60 \mathrm{~cm}$ in height) that contained a hidden platform. The apparatus was used to assess the learning and memory performance of rats. Rats were trained to locate the hidden platform using only distal extra maze cues.

For the navigation test, the tracks of rats were recorded and analyzed by using a camera connected to a video recorder and the EthoVision tracking system (Zhenghua Bio-instruments Co. Ltd., Huaibei, Anhui). Each rat was allowed to search for the platform for 60 seconds, and rest on the platform for 30 seconds once found. Then, the rats were placed back in their respective cages. Rats that failed to find the platform within 60 seconds were manually guided to the platform and placed on the platform for 30 seconds before being returned to their cages. Each rat received four learning trials in the four quadrants $(\mathrm{N}, \mathrm{S}, \mathrm{E}$, and $\mathrm{W})$ per day for five consecutive days. The times spent searching for the platform and the mounting latency were calculated.

On the sixth day, the rats were given the probe test, in which the platform was removed from the pool. Rats were randomly placed into a quadrant and were allowed to swim freely for $60 \mathrm{~s}$. The number of times the rats swarm across the platform in $60 \mathrm{~s}$ was recorded [15].

4.3. Biochemical Assessment. On the seventh day, the rats were sacrificed, and their left and right hippocampus was removed promptly. The right side of the hippocampus was used for hematoxylin and eosin (HE) staining and immunohistochemistry. The left side of the hippocampus was transferred to a $4^{\circ} \mathrm{C}$ ice-cold board for dissection and then used to measure SOD activity and MDA content and to detect the mRNA (reverse transcription polymerase chain 


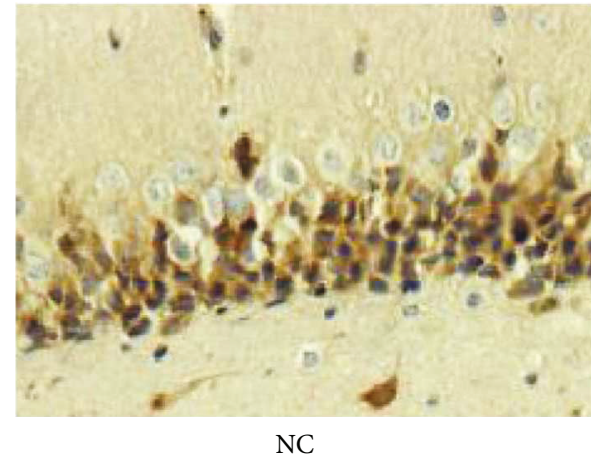

(a)

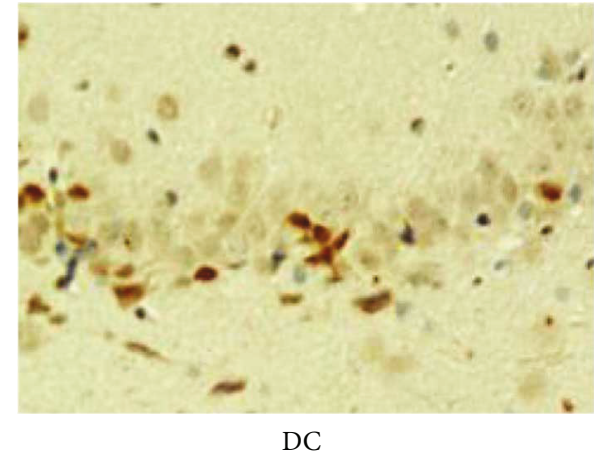

(b)

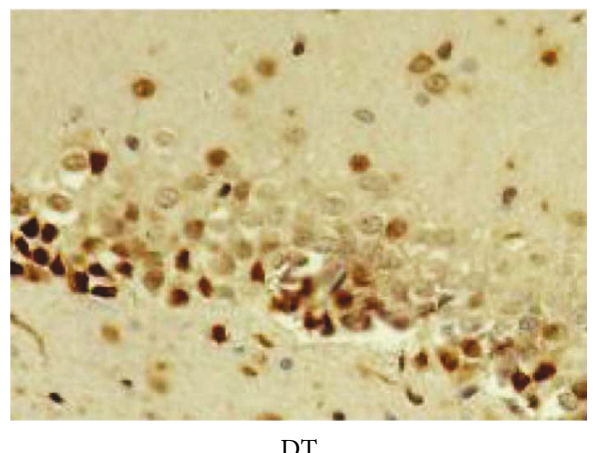

(c)

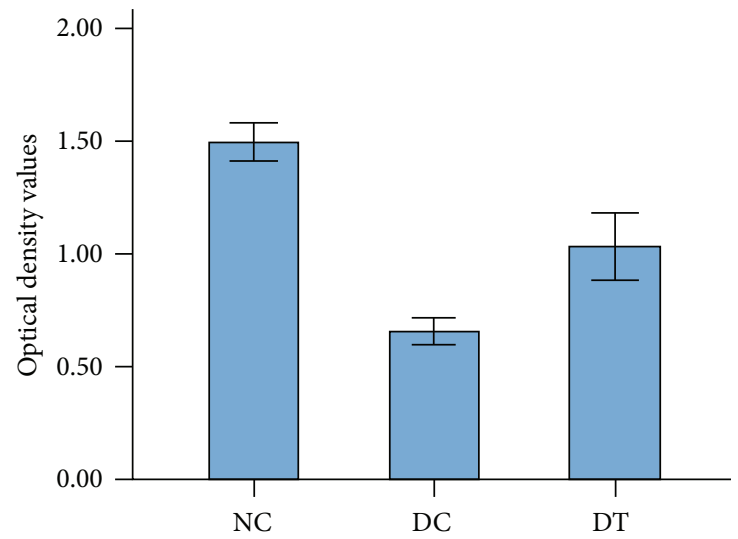

(d)

FIgure 2: The optical density values of Nrf2 in hippocampal CA1 area of each group (IHC, $\times 200)$. Results shown are mean \pm SD $(n=6)$. $P<0.001$ DT versus DC.

reaction, RT-PCR) and protein (Western blot analysis) levels of Nrf2 in both cytoplasm and nucleus.

4.3.1. HE Staining. The syringe containing the sample was kept vertically and still for 10 to 20 minutes, with the needle end pointing downward. The glass slides were cleaned, disinfected with $75 \%$ ethanol, and then dried. One droplet of test solution was added to the glass slide and dried at room temperature. The sample was then fixed in methanol solution for 30 minutes. The methanol solution was removed, and the sample was hydrated with distilled water and subjected to conventional HE staining.
4.3.2. Immunohistochemistry for Nrf2 Expression. Sections were dehydrated in xylene and graded alcohol series. The sections were then rinsed for 5 minutes with tap water and incubated for 30 minutes in $0.3 \% \mathrm{H}_{2} \mathrm{O}_{2}$ in methanol. After, they were washed for 5 minutes with buffer and incubated for 20 minutes in diluted normal serum from the species in which the secondary antibody was obtained. Excess serum from sections was blotted. Then, the sections were incubated for 30 minutes in primary antiserum diluted with buffer. Slides were subsequently washed for 5 minutes with buffer. Sections were incubated for 30 minutes in diluted biotinylated secondary antibody solution, followed by a 5 -minute 


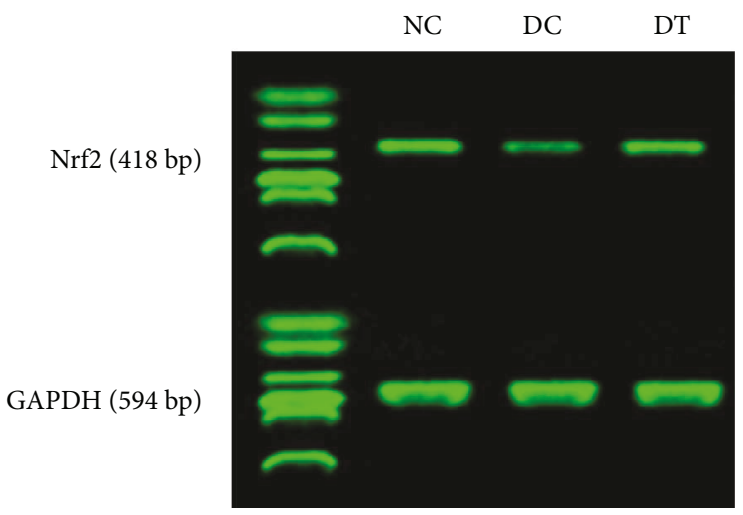

A

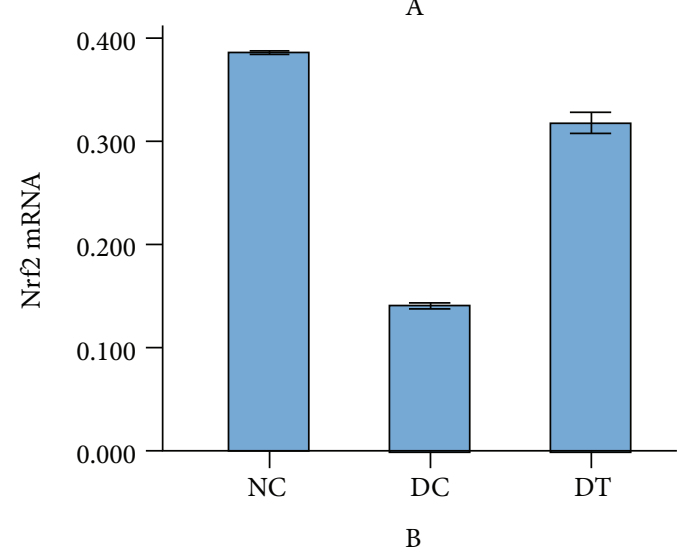

(a)

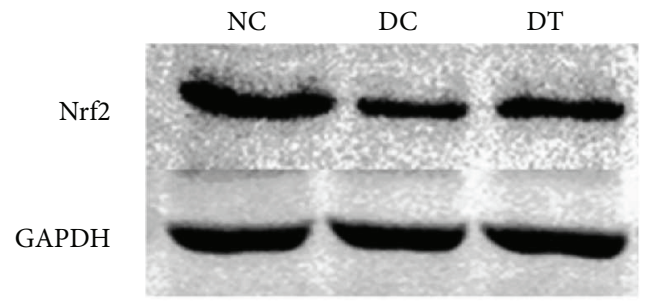

A

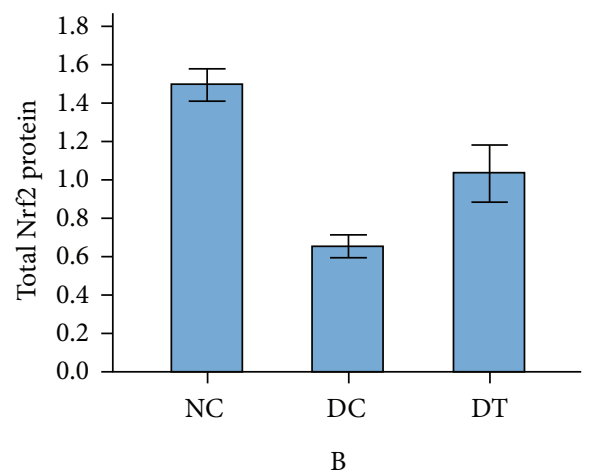

(b)

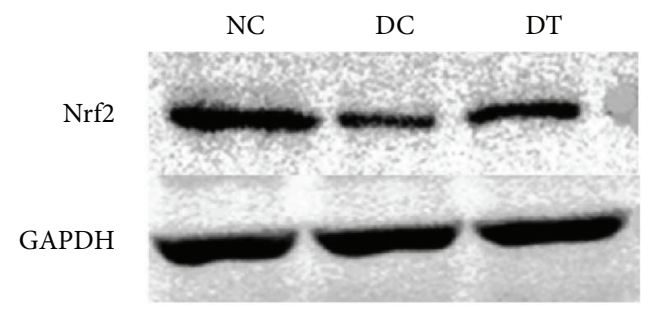

A

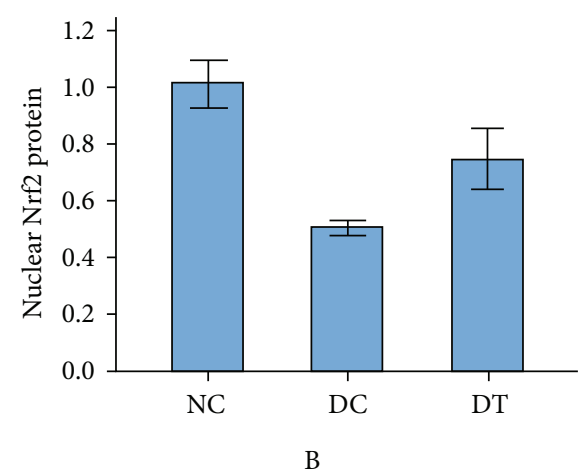

(c)

Figure 3: (a) The expression of total Nrf2 mRNA in different groups. Results shown are mean \pm SD $(n=6), P<0.001$ DT versus DC. (b) The expression of total Nrf2 protein in different groups. Results shown are mean \pm SD $(n=6), P<0.001$ DT versus DC. (c) The expression of nuclear Nrf2 protein in different groups. Results shown are mean $\pm \mathrm{SD}(n=6), P<0.001 \mathrm{DT}$ versus DC.

washing step in buffer. Sections were then incubated for 30 minutes in VECTASTAIN ABC Reagent and washed for another 5 minutes with buffer. Next, sections were incubated in peroxidase substrate solution until the stain developed to the desired intensity. Lastly, sections were rinsed with tap water, counterstained, cleared, and mounted for observation. 


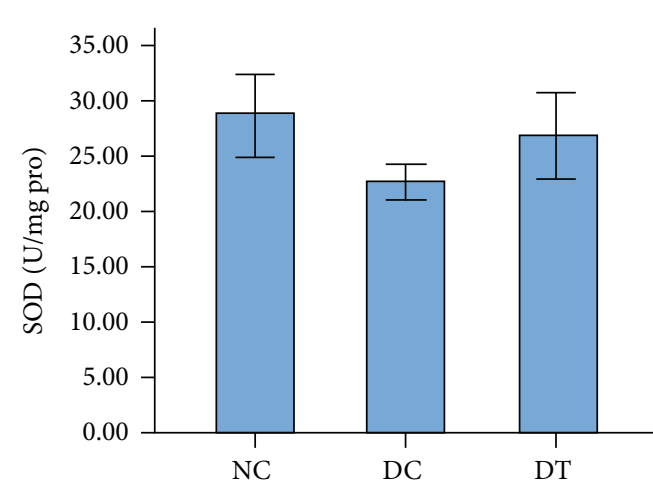

(a)

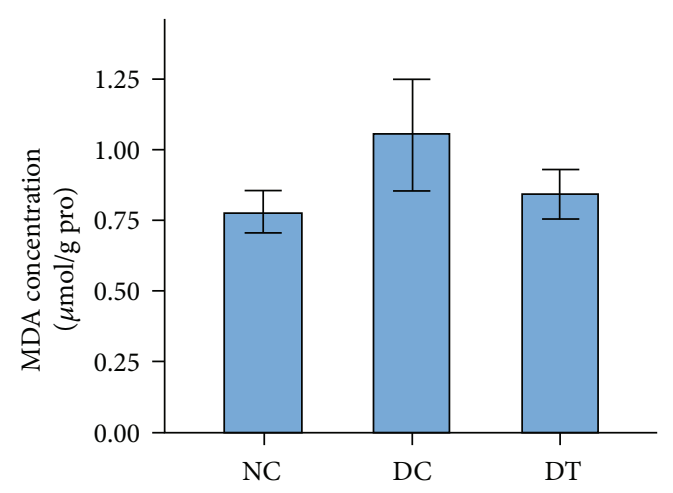

(b)

FIgure 4: (a) The activity of SOD in different groups. Results shown are mean \pm SD $(n=6), P<0.05$ DT versus DC. (b) The MDA concentration in different groups. Results shown are mean $\pm \mathrm{SD}(n=6), P<0.01 \mathrm{DT}$ versus DC.

4.3.3. Quantitative Assessment of Nrf2 $m R N A$ by RT-PCR. Four rats from each group were randomly selected. Total RNA from the hippocampus was isolated using Trizol (Invitrogen Co.), according to the manufacturer's instructions. Reverse transcription was performed using the DEPC (USA Co.). The expression of the housekeeping gene glyceraldehyde-3-phosphate dehydrogenase (GAPDH) mRNA was used as an internal standard. PCR was performed for 30 cycles in an Eppendorf Mastercycler. Denaturing, annealing, and extension reactions were performed at $94^{\circ} \mathrm{C}$ for 30 minutes, $60^{\circ} \mathrm{C}$ for 30 seconds, and $72^{\circ} \mathrm{C}$ for 1 minute, respectively. Then, the PCR products were separated by $1 \%$ agarose gel electrophoresis, stained with GoldView, photographed under ultraviolet illumination, compared with a known standard ladder, and quantified by densitometry using the Bio-1D system. The levels of Nrf2 mRNA were expressed as their respective ratios to GAPDH mRNA.

4.3.4. Western Blotting Analysis for Determining the Nrf2 Protein Levels. Approximately 500-600 mg of tissue homogenate were added to $1 \mathrm{~mL}$ of extraction solution. The mixture was then centrifuged at $12,000 \mathrm{rpm}$ for 5 minutes, at $4^{\circ} \mathrm{C}$, and the supernatant was collected. An equal volume of sample buffer was added to the supernatant on $\chi$ SDS. The sample was then boiled for $5 \mathrm{~min}$ at $100^{\circ} \mathrm{C}$. The same amount of total protein was added to each well, to undergo polyacrylamide gel electrophoresis. The proteins were transferred to a membrane by electric water bath blotting. After the membrane was shaken in a petri dish for 2 hours at room temperature, primary antibody (diluted 1:250 in blocking buffer) [Nrf2 (C-20) is an affinity purified rabbit polyclonal antibody raised against a peptide mapping at the C-terminus of Nrf2 of human origin, Santa Cruz Biotechnology] was added and incubated for 2 hours at room temperature. The membrane was subsequently washed with PBS-Tween three times. After washing, labeled horseradish peroxidase (HRP) secondary antibody (diluted 1:5000 in blocking buffer) [GAPDH(0411) is a mouse monoclonal antibody raised against recombinant GAPDH of human origin] was added, and the petri dish was shaken for 1 hour at room temperature. After washing the membrane sufficiently with PBS-Tween, the membrane was immersed in $\mathrm{DAB}$ color development solution and was let to sit at room temperature for full color development. Then, the membrane was washed with distilled water and immediately transferred to PBS for observation. Lastly, the membrane was photographed and analyzed.

4.3.5. SOD Activity and MDA Content. SOD activity is measured by testing the capacity of pyrogallol to autoxidize. The inhibition of autoxidation of this compound occurs when SOD (Jian Cheng Technology Corporation, China) is present, and the enzymatic activity can be assayed indirectly using a temperature-controlled double-beam spectrophotometer at an absorbance of $420 \mathrm{~nm}$. A 50\% inhibition of pyrogallol autoxidation is defined as $1 \mathrm{U}$ SOD. For testing MDA (Jian Cheng Technology Corporation, China) levels, the samples were mixed with $1 \mathrm{~mL}$ of $10 \%$ trichloroacetic acid and $1 \mathrm{~mL}$ of $0.67 \%$ thiobarbituric acid and then heated in a boiling water bath for 30 minutes. Malondialdehyde equivalents were detected in both tissue and submitochondrial particles of the rats' brain using a spectrophotometer at an absorbance of $532 \mathrm{~nm}$.

4.4. Statistical Analysis. Data were collected and analyzed by the statistical software package SPSS 19.0, which includes one-factor ANOVA with completely randomized design. Repeated measures ANOVA was used to analyze the results of the Morris water maze test. The method of Bonferroni was used to perform pairwise comparisons of the repeatedly measured data from the different measurement times of each treated group, as previously described [22]. As the data passed the normality test $(P>0.10)$, the results were evaluated as the mean \pm standard deviation. Values of $P<0.05$ were considered statistically significant.

\section{Additional Points}

Highlights. (1) This research emphasizes the importance of preventive intervention. (2) The mechanism of diabetic cognitive dysfunction relies on oxidative stress. (3) The prophylactic use of troxerutin can delay the development of diabetic cognitive dysfunction. (4) The prophylactic use of troxerutin can improve the expression of Nrf2 in the hippocampus of STZ diabetic rats. 


\section{Ethical Approval}

This research states that all animal experiments conformed to institutional standards.

\section{Conflicts of Interest}

The authors declare no financial or other conflicts of interest.

\section{References}

[1] K. Itoh, T. Chiba, S. Takahashi et al., "An Nrf2/small Maf heterodimer mediates the induction of phase II detoxifying enzyme genes through antioxidant response elements," Biochemical and Biophysical Research Communications, vol. 236, no. 2, pp. 313-322, 1997.

[2] T. H. Rushmore, M. R. Morton, and C. B. Pickett, "The antioxidant responsive element. Activation by oxidative stress and identification of the DNA consensus sequence required for functional activity," Journal of Biological Chemistry, vol. 266, no. 18, pp. 11632-11639, 1991.

[3] Z. Wang, C. Ji, L. Wu et al., "Tert-butylhydroquinone alleviates early brain injury and cognitive dysfunction after experimental subarachnoid hemorrhage: role of Keap1/Nrf2/ARE pathway," PLoS One, vol. 9, no. 5, article e97685, 2014.

[4] R. Chandran, T. Kim, S. L. Mehta et al., "A combination antioxidant therapy to inhibit NOX2 and activate Nrf2 decreases secondary brain damage and improves functional recovery after traumatic brain injury," Journal of Cerebral Blood Flow \& Metabolism, pp. 1-9, 2017.

[5] S. H. Fan, Z. F. Zhang, Y. L. Zheng et al., "Troxerutin protects the mouse kidney from D-galactose-caused injury through anti-inflammation and anti-oxidation," International Immunopharmacology, vol. 9, no. 1, pp. 91-96, 2009.

[6] C. M. Liu, J. Q. Ma, and Y. Lou, "Chronic administration of troxerutin protects mouse kidney against D-galactoseinduced oxidative DNA damage," Food and Chemical Toxicology, vol. 48, no. 10, pp. 2809-2817, 2010.

[7] Z. F. Zhang, S. H. Fan, Y. L. Zheng et al., "Troxerutin protects the mouse liver against oxidative stress-mediated injury induced by D-galactose," Journal of Agricultural and Food Chemistry, vol. 57, no. 17, pp. 7731-7736, 2009.

[8] J. Lu, D. M. Wu, B. Hu, Y. L. Zheng, Z. F. Zhang, and Y. J. Wang, "NGF-dependent activation of TrkA pathway: a mechanism for the neuroprotective effect of troxerutin in D-galactose-treated mice," Brain Pathology, vol. 20, no. 5, pp. 952-965, 2010.

[9] J. Lu, D. M. Wu, Z. H. Zheng, Y. L. Zheng, B. Hu, and Z. F. Zhang, "Troxerutin protects against high cholesterolinduced cognitive deficits in mice," Brain, vol. 134, no. 3, pp. 783-797, 2011.

[10] Z. Razga, G. Kovacs, N. Bódi, T. P3, and J. R. Nyengaard, "Heterogeneous downregulation of angiotensin II AT1-A and AT1-B receptors in arterioles in STZ-induced diabetic rat kidneys," BioMed Research International, vol. 2014, Article ID 947506, 6 pages, 2014.

[11] T. Baluchnejadmojarad and M. Roghani, "Chronic oral epigallocatechin-gallate alleviates streptozotocin-induced diabetic neuropathic hyperalgesia in rat: involvement of oxidative stress," Iranian Journal of Pharmaceutical Research, vol. 11, no. 4, pp. 1243-1253, 2012.
[12] F. Wang, G. Z. Yang, and S. Liu, "Observational research on type 1 diabetes mellitus rats complicated cognitive dysfunction," in Chinese Medical Association the Seventh Nationwide Young Laboratory Medicine Conference Proceedings, pp. 548549, 2012, http://219.140.69.158/D/Conference_8575243.aspx.

[13] Y. Q. Fu, "Troxerutin pharmacological properties and clinical application profiles," China Pharmaceutical Directory, vol. 10, no. 7, pp. 59-60, 2012.

[14] D. K. Maurya, S. Balakrishnan, V. P. Salvi, and C. K. Nair, "Protection of cellular DNA from $\gamma$-radiation-induced damages and enhancement in DNA repair by troxerutin," Molecular and Cellular Biochemistry, vol. 280, no. 1-2, pp. 57-68, 2005.

[15] C. V. Vorhees and M. T. Williams, "Morris water maze: procedures for assessing spatial and related forms of learning and memory," Nature Protocols, vol. 1, no. 2, pp. 848-858, 2006.

[16] Q. F. Wei, H. L. Lan, and H. Y. Tan, "Research related to diabetes mellitus and cognitive impairment," Medical Review, vol. 16, no. 11, pp. 1696-1699, 2010.

[17] S. H. Wang, X. M. Jia, and H. L. Ke, "Hippocampus and diabetic cognitive dysfunction," Neuroanatomy, vol. 20, no. 6, pp. 637-640, 2004.

[18] F. Atig, M. Raffa, H. B. Ali, A. Saad, and M. Ajina, "Altered antioxidant status and increased lipid peroxidation in seminal plasma of Tunisian infertile men," International Journal of Biological Sciences, vol. 8, no. 1, pp. 139-149, 2012.

[19] J. Lu, D. M. Wu, Y. L. Zheng et al., "Troxerutin counteracts domoic acid-induced memory deficits in mice by inhibiting CCAAT/enhancer binding protein $\beta$-mediated inflammatory response and oxidative stress," The Journal of Immunology, vol. 190, no. 7, pp. 3466-3479, 2013.

[20] H. Y. Cho, S. P. Reddy, and S. R. Kleeberger, "Nrf2 defends the lung from oxidative stress," Antioxidants \& Redox Signaling, vol. 8, no. 1-2, pp. 76-87, 2006.

[21] R. Geetha, B. Yogalakshmi, S. Sreeja, and C. V. Anuradha, "Troxerutin suppresses lipid abnormalities in the heart of high-fat-high-fructose diet-fed mice," Molecular and Cellular Biochemistry, vol. 387, no. 1-2, pp. 123-134, 2014.

[22] H. Qiu, G. Q. Jin, R. F. Jin, and W. K. Zhao, "Analysis of variance of repeated data measured by water maze with SPSS," Journal of Chinese Integrative Medicine, vol. 5, pp. 101-105, 2007. 


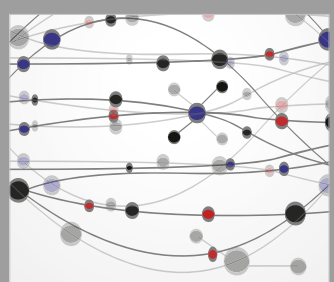

The Scientific World Journal
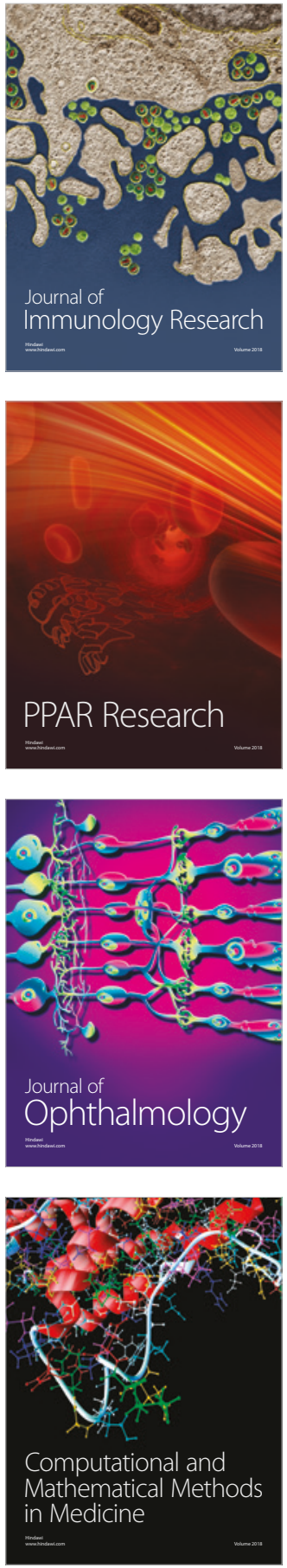

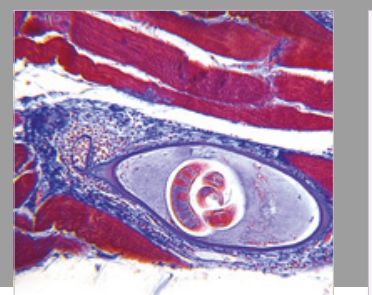

Gastroenterology Research and Practice

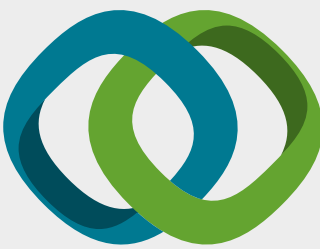

\section{Hindawi}

Submit your manuscripts at

www.hindawi.com
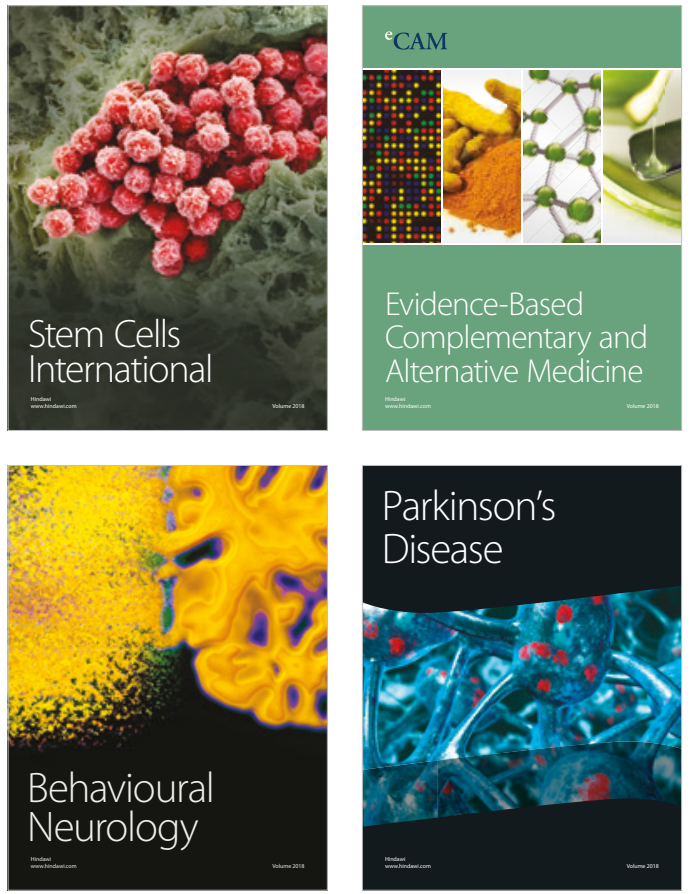

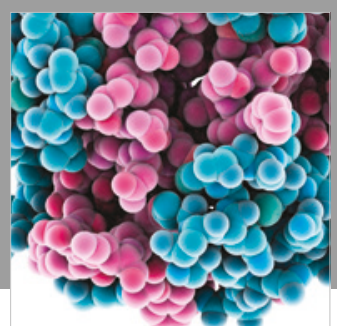

ournal of

Diabetes Research

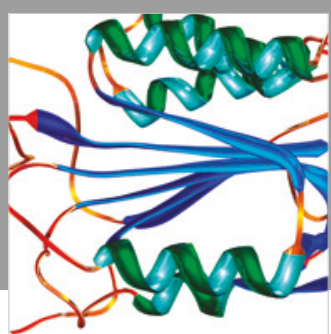

Disease Markers
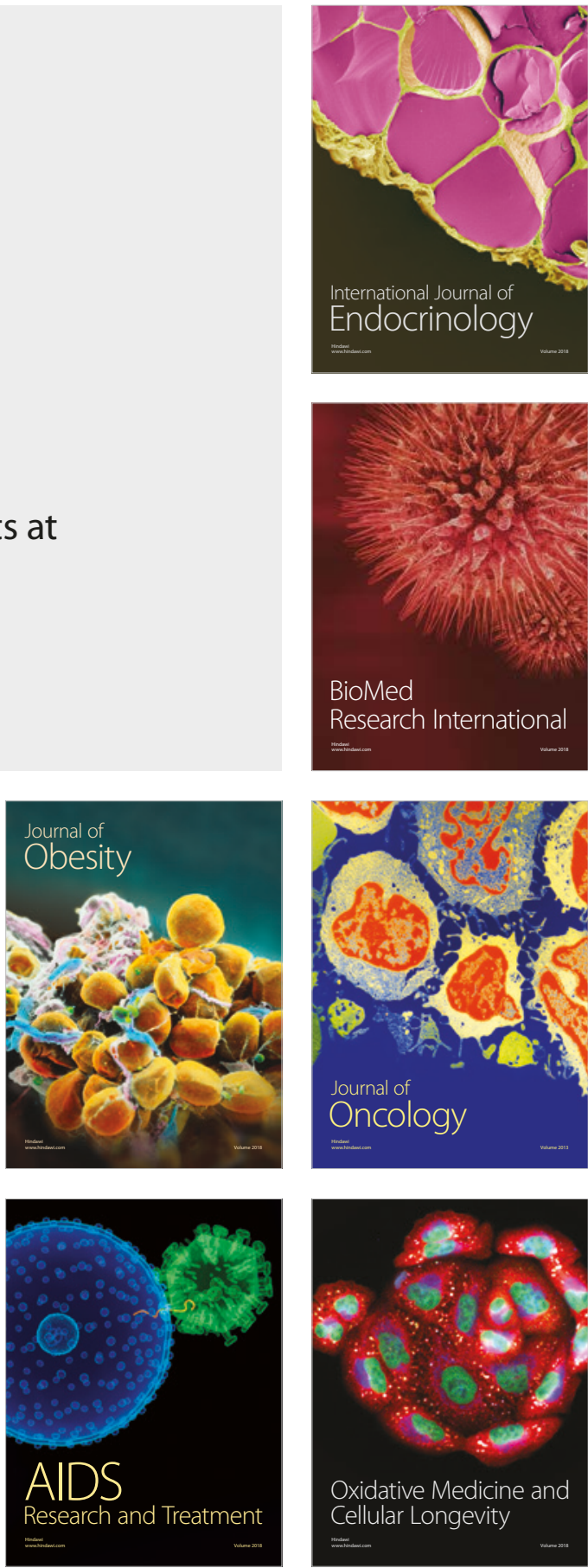